RESEARCH PAPER RP1450

Part of Journal of Research of the National Bureau of Standards, Volume 28, February 1942

\title{
RECTANGULAR PLATE LOADED ALONG TWO ADJACENT EDGES BY COUPLES IN ITS OWN PLANE
}

\author{
By William R. Osgood
}

\section{ABSTRACT}

A. and L. Föppl give an approximate solution for the stresses in the rectangular knee of an L-shaped plate loaded by couples acting on the legs in the plane of the plate. A solution is presented here that is exact in the sense that the equations of equilibrium and the condition of compatibility (for two-dimensional systems) are satisfied at every point. Small self-equilibrated stresses remain along the free edges of the knee.

The stresses in the knees of rigid frames are of considerable interest to engineers, and theoretical analyses, as well as experimental results, may be worth while. Such an analysis for the rectangular knee of a rigid frame has appeared recently. ${ }^{1}$ Part of the analysis involves the stresses produced in the knee by couples acting on the legs in the plane of the rigid frame. The solution used for this condition of loading was not derived in the paper presenting it; and since it appears to be new and not quite obvious, the derivation is presented here for the essentially similar but simpler, special case of a rigid frame without flanges, that is, an L-shaped plate.

Figure 1 illustrates the problem. The linear distribution of stress of the ordinary flexure formula is assumed to apply in the legs at a distance from the knee. At the sharp reentrant corner the stress must become infinite, according to the theory of elasticity, so that the distribution along the inner edges of the knee, shown dotted in the figure, is, according to this theory, probably somewhere between a logarithmic distribution, such as is characteristic of a curved beam, and the linear distribution of a straight beam. Since in practice the theoretically infinite stresses at the corner would be reduced by plastic flow, the final distribution of stress would be represented by a curve with finite ordinates everywhere. In the present paper it is assumed that this distribution can be approximated by a nearly linear distribution, and a polynomial solution is obtained for the stresses in the knee which satisfies the equations of equilibrium and the condition of compatibility (for two-dimensional systems). There is some experimental evidence ${ }^{2}$ that the assumption of a linear distribution is practically justifiable, at least in a rigid frame with flanges running through along the dotted lines in figure 1 . The stresses in the web of the knee are almost surely not appreciably affected by the exact manner of distribution at the junctions between knee and legs. For

\footnotetext{
1 W. R. Osgood, Stresses in a rectangular knee of a rigid frame, J. Research NBS 27, 443 (1941) RP1431.

2 A. H. Stang, Martin Greenspan, and W. R. Osgood, Strength of a riveted steel rigid frame having straight flanges, J. Research NBS 21, 269 (1938) RPi130.
} 
practical purposes of design and analysis of the knee, therefore, the assumption of a nearly linear distribution of stress along the inner edges of the knee, the junctions between knee and legs, seems sufficiently accurate.

A. and L. Föppl ${ }^{3}$ give a solution for the knee in which they assume a strictly linear distribution along the inner edges. Their solution

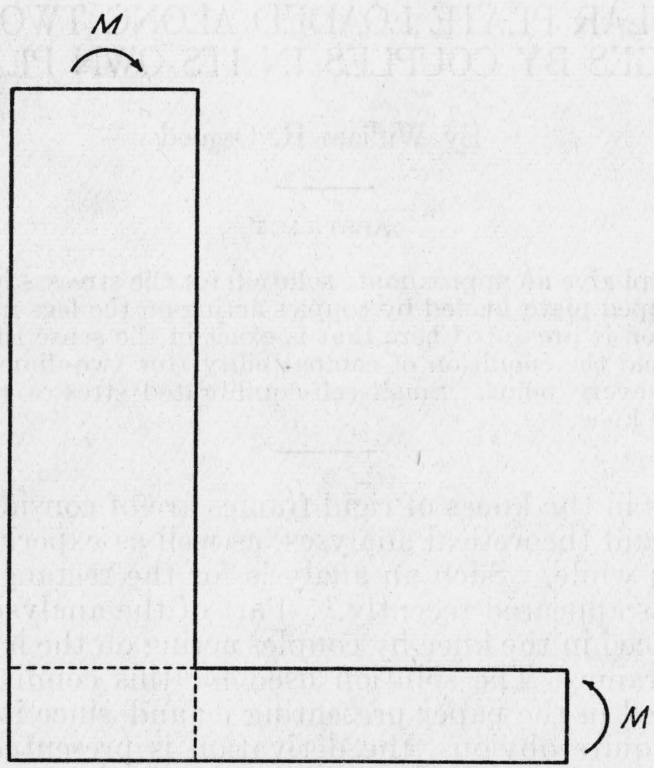

FigURE 1.-L-shaped plate loaded by couples.

satisfies the boundary conditions and the equations of equilibrium at every point, but it does not satisfy the condition of compatibility. The solution proposed here may be built up by judiciously superposing known solutions for cantilever beams. The equations of equilibrium

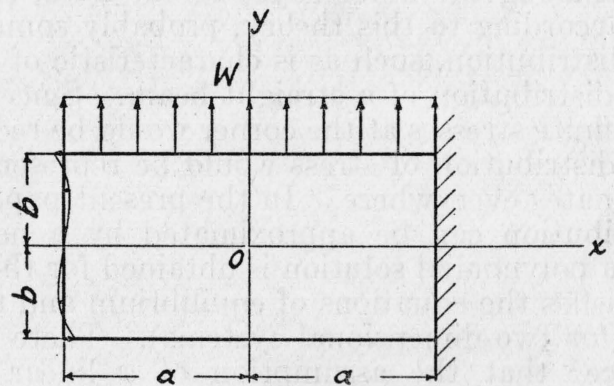

Figure 2.-Cantilevered plate acted on by uniformly distributed load.

and the condition of compatibility are satisfied at every point, but small self-equilibrated stresses remain on the outer edges of the knee. Since these boundary stresses are relatively small and their effect can be readily judged, it seems preferable to sacrifice the boundary conditions rather than the condition of compatibility, the consequences of

3 Drang und Zwang 1, 340 (1924). 
the latter sacrifice not being so readily apparent. (Rather large selfequilibrated stresses do in fact occur. See footnote 2.)

We proceed now to the proposed solution. The solution for the uniformly loaded cantilever beam or cantilevered plate of thickness $t$ shown in figure 2 is ${ }^{4}$

$$
\left.\begin{array}{rl}
\sigma_{x} & =-\frac{3 W a}{8 b^{2} t}\left(1+\frac{x}{a}\right)^{2} \frac{y}{b}-\frac{W}{4 a t} \frac{y}{b}\left(\frac{3}{5}-\frac{y^{2}}{b^{2}}\right), \\
\sigma_{y} & =\frac{W}{4 a t}\left(1-\frac{y}{2 b}\right)\left(1+\frac{y}{b}\right)^{2}, \\
\tau_{x y} & =-\frac{3 W}{8 b t}\left(1+\frac{x}{a}\right)\left(1-\frac{y^{2}}{b^{2}}\right) .
\end{array}\right\}
$$

The solution for the cantilevered plate of thickness $t$ carrying a uniformly varying total load $W$ as shown in figure 3 is ${ }^{5}$

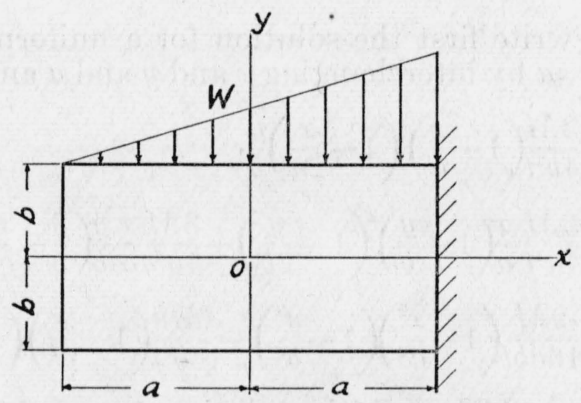

FigURE 3.-Cantilevered plate acted on by uniformly varying load.

$$
\left.\begin{array}{rl}
\sigma_{x} & =\frac{W a}{8 b^{2} t}\left(1+\frac{x}{a}\right)^{3} \frac{y}{b}+\frac{W}{4 a t}\left(1+\frac{x}{a}\right) \frac{y}{b}\left(\frac{3}{5}-\frac{y^{2}}{b^{2}}\right), \\
\sigma_{y} & =-\frac{W}{4 a t}\left(1+\frac{x}{a}\right)\left(1-\frac{y}{2 b}\right)\left(1+\frac{y}{b}\right)^{2}, \\
\tau_{x y} & =\frac{3 W}{16 b t}\left(1+\frac{x}{a}\right)^{2}\left(1-\frac{y^{2}}{b^{2}}\right)+\frac{W b}{16 a^{2} t}\left(1-\frac{y^{2}}{b^{2}}\right)\left(\frac{1}{5}-\frac{y^{2}}{b^{2}}\right)
\end{array}\right\}
$$

If we superpose these two solutions, we obtain the solution for a plate loaded by couples $M=W a / 3$ on the two edges $x=a$ and $y=b$, figure 4:

$$
\left.\begin{array}{rl}
\sigma_{x} & =-\frac{3 M}{4 b^{2} t}\left(1+\frac{x}{a}\right)^{2}\left(1-\frac{x}{2 a}\right) \frac{y}{b}+\frac{3 M}{4 a^{2} t} \frac{x}{a} \frac{y}{b}\left(\frac{3}{5}-\frac{y^{2}}{b^{2}}\right), \\
\sigma_{y} & =-\frac{3 M}{4 a^{2} t} \frac{x}{a}\left(1+\frac{y}{b}\right)^{2}\left(1-\frac{y}{2 b}\right), \\
\tau_{x y} & =-\frac{9 M}{16 a b t}\left(1-\frac{x^{2}}{a^{2}}\right)\left(1-\frac{y^{2}}{b^{2}}\right)+\frac{3 M b}{16 a^{3} t}\left(1-\frac{y^{2}}{b^{2}}\right)\left(\frac{1}{5}-\frac{y^{2}}{b^{2}}\right) \cdot
\end{array}\right\}
$$

Along the edge $y=b$ the forces are uniformly varying but not along the edge $x=a$. This "unsymmetrical" condition is not of much

4 R. V. Southwell, Theory of Elasticity, p. 377 (1936).

o S. Timoshenko, Theory of Elasticity, p. 42 (1934). 
interest. Moreover, for large values of $b / a$ the self-equilibrated normal stresses (second term on right-hand side of expression for $\sigma_{x}$ ) at $x=-a$ become relatively large. These objections may be overcome

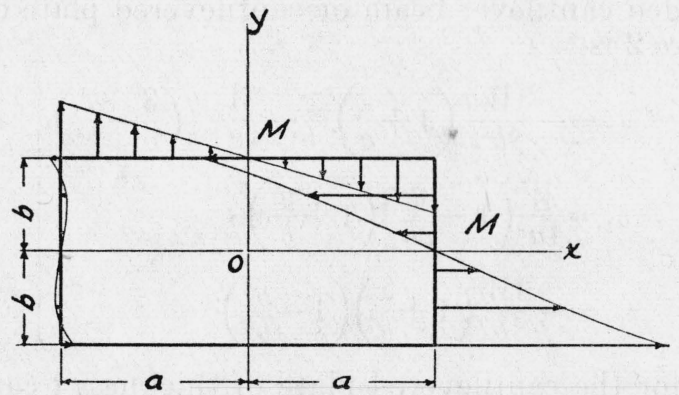

FiguRe 4.-Rectangular plate loaded unsymmetrically by couples.

as follows. We write first the solution for a uniformly varying load along the edge $x=a$ by interchanging $x$ and $y$ and $a$ and $b$ in solution 3:

$$
\left.\begin{array}{rl}
\sigma_{x} & =-\frac{3 M}{4 b^{2} t}\left(1+\frac{x}{a}\right)^{2}\left(1-\frac{x}{2 a}\right) \frac{y}{b}, \\
\sigma_{y} & =-\frac{3 M}{4 a^{2} t} \frac{x}{a}\left(1+\frac{y}{b}\right)^{2}\left(1-\frac{y}{2 b}\right)+\frac{3 M}{4 b^{2} t} \frac{x}{a} \frac{y}{b}\left(\frac{3}{5}-\frac{x^{2}}{a^{2}}\right), \\
\tau_{x y} & =-\frac{9 M}{16 a b t}\left(1-\frac{x^{2}}{a^{2}}\right)\left(1-\frac{y^{2}}{b^{2}}\right)+\frac{3 M a}{16 b^{3} t}\left(1-\frac{x^{2}}{a^{2}}\right)\left(\frac{1}{5}-\frac{x^{2}}{a^{2}}\right)
\end{array}\right\}
$$

Now if we replace $M$ by $\alpha M$ in solution 3 and $M$ by $(1-\alpha) M$ in solution 4 and then superpose these two solutions, we obtain another solution for a plate loaded by couples $M$ on adjacent edges; and by varying $\alpha$ the distribution of the loads may be made to vary between that required by solution 3 and that required by solution 4:

$$
\begin{aligned}
\sigma_{x}= & -\frac{3 M}{4 b^{2} t}\left(1+\frac{x}{a}\right)^{2}\left(1-\frac{x}{2 a}\right) \frac{y}{b}+\frac{3 \alpha M}{4 a^{2} t} \frac{x}{a} \frac{y}{b}\left(3-\frac{y^{2}}{b^{2}}\right) \\
\sigma_{\nu}=- & \frac{3 M}{4 a^{2} t} \frac{x}{a}\left(1+\frac{y}{b}\right)^{2}\left(1-\frac{y}{2 b}\right)+\frac{3(1-\alpha) M}{4 b^{2} t} \frac{x}{a} \frac{y}{b}\left(\frac{3}{5}-\frac{x^{2}}{a^{2}}\right) \\
\tau_{x y}=- & \frac{9 M}{16 a b t}\left(1-\frac{x^{2}}{a^{2}}\right)\left(1-\frac{y^{2}}{b^{2}}\right)+\frac{3 M}{16 t}\left[\frac{\alpha b}{a^{3}}\left(1-\frac{y^{2}}{b^{2}}\right)\left(\frac{1}{5}-\frac{y^{2}}{b^{2}}\right)\right. \\
& \left.+\frac{(1-\alpha) a}{b^{3}}\left(1-\frac{x^{2}}{a^{2}}\right)\left(\frac{1}{5}-\frac{x^{2}}{a^{2}}\right)\right]
\end{aligned}
$$

If we wish to keep the ratio of the self-equilibrated normal stress to the remaining normal stress the same for equal values of $x / a$ and $y / b$, inspection of the first two of eq 5 shows that we must have

$$
\frac{b^{2} \alpha}{a^{2}}=\frac{(1-\alpha) a^{2}}{b^{2}}
$$

or

$$
\alpha=\frac{a^{4}}{a^{4}+b^{4}} .
$$


With this value of $\alpha$ eq 5 become

$$
\left.\begin{array}{rl}
\sigma_{x}= & -\frac{3 M}{4 b^{2} t}\left(1+\frac{x}{a}\right)^{2}\left(1-\frac{x}{2 a}\right) \frac{y}{b}+\frac{3 M a^{2}}{4\left(a^{4}+b^{4}\right) t} \frac{x}{a} \frac{y}{b}\left(\frac{3}{5}-\frac{y^{2}}{b^{2}}\right), \\
\sigma_{y}= & -\frac{3 M}{4 a^{2} t} \frac{x}{a}\left(1+\frac{y}{b}\right)^{2}\left(1-\frac{y}{2 b}\right)+\frac{3 M b^{2}}{4\left(a^{4}+b^{4}\right) t} \frac{x}{a} \frac{y}{b}\left(\frac{3}{5}-\frac{x^{2}}{a^{2}}\right), \\
\tau_{x y}= & -\frac{9 M}{16 a b t}\left(1-\frac{x^{2}}{a^{2}}\right)\left(1-\frac{y^{2}}{b^{2}}\right) \\
& +\frac{3 M a b}{16\left(a^{4}+b^{4}\right) t}\left[\left(1-\frac{x^{2}}{a^{2}}\right)\left(1-\frac{x^{2}}{a^{2}}\right)+\left(1-\frac{y^{2}}{b^{2}}\right)\left(1-\frac{y^{2}}{b^{2}}\right)\right] .
\end{array}\right\}
$$

This solution is symmetrical, and the self-equilibrated stresses, represented by the second of each of the two terms on the right-

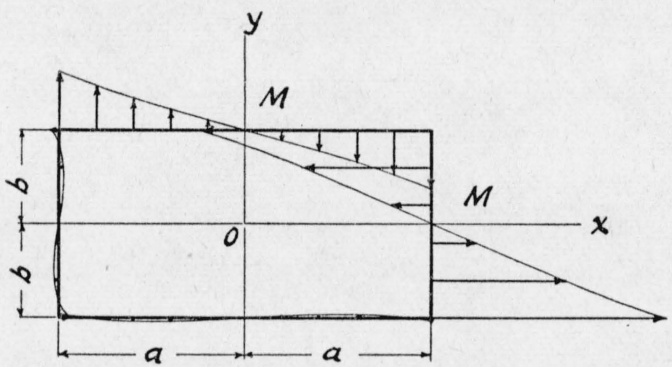

FIGURE 5.-Rectangular plate loaded symmetrically by couples.

hand sides, can never become excessive. At $x=a, y=b$, for example, the second term of $\sigma_{x}$ or of $\sigma_{y}$ is equal to $a^{2} b^{2} /\left[5\left(a^{4}+b^{4}\right)\right]$ times the first term. The maximum value this fraction can have is $1 / 10$, when $a=b$. Figure 5 shows the distribution of stress along the boundary for $a / b=2$. The self-equilibrated shearing stresses are too small to appear in the drawing.

Washington, September 17, 1941. 